Series A

I. MATHEMATICA

526

\title{
THE BOUNDARY MAPPING INDUCED BY AN ISOMORPHISM OF COVERING GROUPS
}

BY

TUOMAS SORVALI

H E L S I N K I 1972

S U O M A L A I E N T IEDEAKA TEM I A 
Copyright (C) 1972 by

Academia Scientiarum Fennica

ISBN 951-41-0067-0

Communicated 13 October 1972 by OLLI Lehto 


\section{Acknowledgements}

I am greatly indebted to my teachє $\mathrm{r}$, Professor O. Leнто, for suggesting the subject of this investigation and for his unfailing interest and valuable advice throughout the course of this work.

I wish also to express my gratitude to the Academy of Finland, to Case Western Reserve University and to the University of Helsinki for financial support.

To Suomalainen Tiedeakatemia (Fimnish Academy of Science) I am grateful for accepting this work to be published in the series of their annals.

Siilinjärvi, July 1972

TuOmas Sorvali 


\section{Introduction}

Every Riemann surface with the unit disk $D$ as a universal covering surface can be represented as a quotient space $D / G$, where the covering group $G$, consisting of conformal self-mappings of $D$, is discontinuous and fixed point free. If the fixed points of $G$ are dense in the unit circle $K$, the group $G$ is said to be of the first kind.

In this paper we consider isomorphisms $j$ between two covering groups $G$ and $G^{\prime}$ of the first kind, with the following property: There exists a homeomorphism $\varphi: K \rightarrow K$ sending the attracting fixed point of every $g \in G$ to the attracting fixed point of $j(g)$. We call $\varphi$ the boundary mapping of $j$. It induces the isomorphism $j$ on $K$, i.e., $\varphi \circ g=j(g) \circ \varphi$.

An isomorphism $j$ has a boundary mapping if and only if there exists a homeomorphism $f: D \rightarrow D$ inducing $j$ in $D$. This was proved by Nielsen [17] in the case of compact surfaces $D / G$ and $D / G^{\prime}$, and by Fenchel and Nielsen [9] if $G$ is finitely generated. In the general case, the simultaneous existence of $\varphi$ and $f$ was recently proved by Tukia [20], and it also follows from earlier unpublished results of Marden [16].

After some preliminary considerations in $\S \S 1$ and 2 , we summarize and complement results of Nielsen, Marden and Tukia in §3. Isomorphisms not possessing a boundary mapping are analyzed and an example of the situation is given.

In $\S 4$ we introduce the dilatation of an isomorphism defined as follows: If $x(g)$ denotes the multiplier of $g \in G$, then the dilatation $\delta(j)$ of $j$ is the smallest number $a \geq 1$ for which $\varkappa(g)^{1 / a} \leq \varkappa(j(g)) \leq \varkappa(g)^{a}$ holds for all $g \in G$. We prove that if $\delta(j)=1$, then the boundary mapping $\varphi$ of $j$ exists and preserves cross ratios, i.e., $j$ is induced by a Möbius transformation. Secondly, if $j$ is induced by a $K$-quasiconformal mapping $f$, then $\delta(j) \leq K$. Our third result connects $\delta(j)$ and the quasisymmetry of $\varphi:$ If $G$ and $G^{\prime}$ act in the upper half-plane and $\varphi: R \rightarrow R$ is $\lambda$-quasisymmetric, then $\delta(j) \leq \log 2 / \log (1+1 / \lambda)$. The section is concluded by an example of an isomorphism possessing $\varphi$ and satisfying $\delta(j)=\infty$.

Section 5 deals with isomorphisms with a special boundary mapping. We prove that if $\varphi$ and $\phi^{-1}$ are locally Lipschitzian, then $j$ is induced 
by a Möbius transformation. Remarks are made on isomorphisms with quasisymmetric boundary mappings, recently treated by Lehto [14], and some conjectures and open problems are mentioned.

\section{§1. The universal covering surface}

1. Let $C$ denote the complex plane, $\hat{C}$ the extended plane $C \cup\{\infty\}$, $R$ the set of real numbers and $\hat{R}=R \cup\{-\infty, \infty\}$. Set $D=\{z \in C$ $|z|<1\}, K=\{z \in C|| z \mid=1\}, I=\{t \in R \mid 0 \leq t \leq 1\}$ and $H=\{z \in C \mid$ $\operatorname{Im} z>0\}$. The complex conjugate of $z=x+i y$ is denoted by $\bar{z}=x$ $-i y$.

If $A$ is a subset of a topological space, the closure of $A$ is denoted by $\mathrm{Cl} A$ and the boundary by $\mathrm{Bd} A$. Let $A \backslash B$ mean the complement of $B$ in $A$.

A universal covering surface of a Riemann surface $S$ is a pair $(\tilde{S}, \pi)$ satisfying the following conditions:

(i) $\tilde{S}$ is a simply connected Riemann surface.

(ii) $\pi: \tilde{S} \rightarrow S$ is a locally conformal mapping.

(iii) If $\gamma: I \rightarrow S$ is a path and $\pi(\tilde{p})=\gamma(0)$. there exists a path $\tilde{\gamma}: I \rightarrow \tilde{S}$ such that $\tilde{\gamma}(0)=\tilde{p}$ and $\pi \circ \tilde{\gamma}=\gamma$.

The path $\tilde{\gamma}$ in (iii) is referred to as the lifting of $\gamma$ from the point $\tilde{p}$ over $\gamma(0)$.

By the Riemann mapping theorem, $\tilde{S}$ is conformally equivalent to one of the canonical regions $\hat{C}, C$ or $D$. The case $\tilde{S}=\hat{C}$ is possible if and only if $S$ is conformally equivalent to $\hat{C}$ : and $\tilde{S}=C$ occurs if and only if $S$ is conformally equivalent to $C$ or $C \backslash\{0\}$, or $S$ is a compact Riemann surface of genus one (i.e. a torus). The special cases will be excluded in the following: If not otherwise stated, we shall always assume that $\tilde{S}$ is conformally equivalent to $D$.

2. Throughout the paper $D^{\prime}: D^{\prime \prime}$ will denote arbitrary disks (halfplanes included).

Let $G$ be a group of Möbius transformations acting in $D^{\prime}$, i.e., $g\left(D^{\prime}\right)$ $=D^{\prime}$ for all $g \in G$. A set $A \subset D^{\prime}$ is called a fundamental set of $G$ if it has the following properties:

(i) $g(A) \cap A=\varnothing$ for all $g \in G \backslash\{i d\}$.

(ii) $D^{\prime}=\bigcup_{g \in G} g(A)$.

By the axiom of choic e, $G$ has fundamental sets if and only if $G$ acts freely in $D^{\prime}$. i.e., the fixed points of $G$ lie in $\mathrm{Bd} D^{\prime}$. 
If $G$ has fundamental sets containing interior points, then $G$ is called a covering group of $D^{\prime}$. It follows immediately that a covering group is denumerable.

A region $B \subset D^{\prime}$ is called a fundamental domain of $G$ if $G$ has a fundamental set $A$ such that $B \subset A \subset \mathrm{Cl} B$. It follows that every covering group has fundəmental domains (cf. 3.3).

3. Let $\left(D^{\prime}, \pi\right)$ be the universal covering surface of a Riemann surface $S$. A covering transformation of $\left(D^{\prime}, \pi\right)$ over $S$ is a conformal mapping $g: D^{\prime} \rightarrow D^{\prime}$ (i.e., a Möbius transformation fixing $D^{\prime}$ ) satisfying $\pi \circ g=\pi$. The group of covering transformations of $\left(D^{\prime}: \pi\right)$ over $S$ is a covering group of $D^{\prime}$.

On the other hand, if a covering group $G$ of $D^{\prime}$ is given, then $D^{\prime} / G$ is a Riemann surface and $G$ is the group of covering transformations of $\left(D^{\prime}, \pi\right)$ over $D^{\prime} / G$, where $\pi: D^{\prime} \rightarrow D^{\prime} / G$ is the canonical projection.

Let $G$ and $G^{\prime}$ be covering groups of $D^{\prime}$ and $D^{\prime \prime}$, respectively. The groups $G$ and $G^{\prime}$ are conjugate if there exists a Möbius transformation $h: D^{\prime} \rightarrow D^{\prime \prime}$ such that $G^{\prime}==h G h^{-1}$. The Riemann surfaces $D^{\prime} / G$ and $D^{\prime \prime} / G^{\prime}$ are conformally equivalent if and only if $G$ and $G^{\prime}$ are conjugate. (For details, see [3].)

4. For later reference we recall here some basic properties of Möbius transformations.

Suppose that $g$ is a Möbius transformation fixing a disk or a halfplane $D^{\prime}$. Then, by the reflection principle, the fixed points $x$ and $y$ of $g$ either lie on $\mathrm{Bd} D^{\prime}$ or are symmetric points with respect to $\operatorname{Bd} D^{\prime}$. If in the former case $x \neq y, g$ is called hyperbolic, and if $x=y, g$ is parabolic. In the latter case $g$ is elliptic. Since a covering group of $D^{\prime}$ acts freely in $D^{\prime}$, it contains hyperbolic and parabolic transformations only.

For a hyperbolic transformation $g$, let $P(g)$ and $N(g)$ denote the attracting and the repelling fixed point of $g$, i.e., for every $z \in \hat{C} \backslash\{x, y\}$

$$
P(g)=\lim _{n \rightarrow \infty} g^{n}(z), \quad N(g)=\lim _{n \rightarrow \infty} g^{-n}(z) .
$$

Suppose that $P(g) \neq \infty \neq N(g)$. Then there is a real number $x>1$ such that

$$
\frac{g(z)-N(g)}{g(z)-P(g)}=\varkappa \frac{z-N(g)}{z-P(g)}
$$

If $P(g)=\infty$, then

$$
g(z)-N(g)=\varkappa(z-N(g))
$$


and if $N(g)=\infty$, then

$$
g(z)-P(g)=(z-P(g)) / \varkappa .
$$

The real number $\varkappa=\varkappa(g)$ is called the multiplier of $g$.

Two Möbius transformations $g$ and $g^{*}$ are conjugate if there is a Möbius transformation $h$ such that $g^{*}=h \circ g \circ h^{-1}$. Then $g^{*}$ is hyperbolic if and only if $g$ is, and it follows that

$$
x(g)=x\left(g^{*}\right) \text {. }
$$

If a hyperbolic transformation $g$ is given in the form

$$
g(z)=\frac{a z+b}{c z+d}, \quad a d-b c=1,
$$

an elementary calculation shows that

$$
(a+d)^{2}-2=x(g)+1 / x(g) .
$$

Hence $a+d$ is always real. The number $\chi(g)=|a+d|$ is called the trace of $g$. By (1.3) we have

$$
\chi(g)=\varkappa(g)^{1 / 2}+\chi(g)^{-1 / 2} .
$$

It follows that $\chi(g)>2$, and $\chi\left(g_{1}\right)=\chi\left(g_{2}\right)$ if and only if $\%\left(g_{1}\right)=*\left(g_{2}\right)$. Thus by (1.2)

$$
\chi(g)=\chi\left(g^{*}\right) \text {. }
$$

If $g$ is parabolic, then $a+d= \pm 2$. Therefore it is natural to define $\chi(g)=2$ and $\varkappa(g)=1$. Then formulae (1.2)-(1.5) automatically hold also in this case. If $x$ is the only fixed point of $g$, then

$$
x=\lim _{n \rightarrow \pm \infty} g(z) \text {. }
$$

Therefore we set $P(g)=N(g)=x$.

The cross ratio $\left(z_{1}, z_{2}, z_{3}, z_{4}\right)$ of four distinct points $z_{1}, \ldots, z_{4}$ of $\hat{C}$ is defined as the image of $z_{1}$ under the Möbius transformation which carries $z_{2}, z_{3}, z_{4}$ to the points $1,0, \infty$, respectively. It follows that there is a Möbius transformation carrying $z_{1}, \ldots, z_{4}$ to $u_{1}, \ldots, w_{4}$ if and only if $\left(z_{1}, z_{2}, z_{3}, z_{4}\right)=\left(w_{1}, w_{2}, w_{3}, w_{4}\right)$. Consequently the points $z_{1}, \ldots, z_{4}$ lie on a circle or a straight line if and only if $\left(z_{1}, z_{2}, z_{3}, z_{4}\right)$ is real.

5. We conclude this preliminary section with some remarks concerning quasiconformal mappings.

Let $\varphi: \hat{R} \rightarrow \widehat{R}$ be an increasing bijection and define 


$$
\begin{gathered}
w(x+i y)=\frac{1}{2} \int_{0}^{1}(\varphi(x+t y)+\varphi(x-t y)) d t+ \\
\frac{i}{2} \int_{0}^{1}(\varphi(x+t y)-\varphi(x-t y)) d t .
\end{gathered}
$$

It is well known that $w: \hat{C} \rightarrow \hat{C}$ is a homeomorphism with the following properties (see [8] and [15]):

(i) $w \mid \hat{R}=\varphi$,

(ii) $w(H)=H$,

(iii) $w(\bar{z})=\overline{w(z)}$,

(iv) $w \mid H$ is a diffeomorphism.

It follows from (iv) that $w \mid H$ is locally quasiconformal, i.e., if $A$ is a region such that $\mathrm{Cl} A \subset H$ is compact, then $w \mid A$ is quasiconformal.

Suppose that $\varphi$ is quasisymmetric on an open interval $I_{0} \subset R$, i.e., there exists a real number $\lambda \geq 1$ such that

$$
1 / \lambda \leq \frac{\varphi(x+t)-\varphi(x)}{\varphi(x)-\varphi(x-t)} \leq \lambda
$$

holds for all $x$ and $t, x \pm t \in I_{0}$. If $I_{1} \subset I_{0}$ is a closed interval, then $I_{1}$ has a neighborhood $U$ such that $w \mid U$ is quasiconformal (see [15], p. 88). If (1.7) holds for all $x$ and $t$, then $w: \hat{C} \rightarrow \hat{C}$ is quasiconformal. Conversely, if a quasiconformal mapping $w: H \rightarrow H$ is given, then $w$ can be extended to a homeomorphism $w: H \cup R \cup\{\infty\} \rightarrow H \cup R \cup\{\infty\}$, and if $w(\infty)=\infty$, then $w \mid R$ is quasisyminetric.

6. To generalize the last remark in 5, consider the following situation: Let $w_{0}: H \cup R \cup\{\infty\} \rightarrow H \cup R \cup\{\infty\}$ be a homeomorphism such that $w_{0}(\infty)=\infty$ and $w_{0} \mid R$ is increasing. Suppose that $D^{\prime}$ is an open disk such that $I_{0}=D^{\prime} \cap R \neq \varnothing$ and $w_{0} \mid D^{\prime} \cap H$ is quasiconformal. Let $I_{1}, \mathrm{Cl} I_{1} \subset I_{0}$, be an open interval. We prove that $w_{0} \mid I_{1}$ is quasisymmetric. First, it follows that the interval $w_{0}\left(I_{0}\right)$ is a free boundary arc of $w_{0}\left(D^{\prime} \cap H\right)$. Thus, if we define $w_{0}(\bar{z})=\overline{w_{0}(z)}$, the mapping $w_{0}$ is quasiconformal in a region containing $\mathrm{Cl} I_{1}$. Since $\mathrm{Cl} I_{1}$ is compact, there exists a quasiconformal mapping $w_{1}: \hat{C} \rightarrow \hat{C}$ such that $w_{1} \mid I_{1}=$ $w_{0} \mid I_{1}\left([15]\right.$, II. 8.1). If $x \in I_{1}, t>0$ and $x \pm t \in I_{1}$, then $(x, \infty$, $x+t, x-t)=-1$. Therefore, by the quasi-invariance of cross ratios under a quasiconformal mapping, there exists a $\lambda \geq 1$ which does not depend on the choice of $x$ and $t$, such that

$$
1 / \lambda \leq\left|\left(w_{1}(x), w_{1}(\infty), w_{1}(x+t), w-_{1}(x t)\right)\right| \leq \lambda .
$$


(This follows from Theorem 3.2 in [1].) If $w_{1}(\infty)=\infty$, there is nothing more to prove. If not, then

$$
\begin{aligned}
& \left|\left(w_{1}(x), w_{1}(\infty), w_{1}(x+t), w_{1}(x-t)\right)\right|= \\
& \frac{w_{0}}{w_{0}} \frac{(x+t)-w_{0}(x)}{(x)-w_{0}(x-t)} \cdot\left|\frac{w_{1}(\infty)-w_{1}(x-t)}{w_{1}(\infty)-w_{1}(x+t)}\right| .
\end{aligned}
$$

By the compactness of $\mathrm{Cl} I_{1},\left|w_{1}(\infty)-w_{1}(x-t)\right| \cdot\left|w_{1}(\infty)-w_{1}(x+t)\right|^{-1}$ is bounded away from 0 and $\infty$, and the proof is complete.

If we define $w$ by (1.6) with the boundary values $\varphi=w_{0} \mid \hat{R}$, then it follows that $w$ is quasiconformal in a neighborhood of $I_{1}$.

7. Applying the considerations in 6 , we prove the following lemma needed in $\S 2$.

Lemma 1.1. Let $f: D \rightarrow D$ be a sense-preserving homeomorphism and $D^{\prime}$ an open disk such that $\mathrm{Cl} D^{\prime} \subset D$. Then there is a homeomorphism $f^{\prime}: D \rightarrow D$ having the following properties:

( i) $f^{\prime}=f$ in $D \backslash D^{\prime}$,

(ii) $f^{\prime}$ is locally quasiconformal in $D^{\prime}$,

(iii) if $f$ is quasiconformal in a neighborhood of a point of $\operatorname{Bd} D^{\prime}$, then the same holds true of $f^{\prime}$,

(iv) if $f$ is quasiconformal in a neighborhood of every point of $\operatorname{Bd} D^{\prime}$, then $f^{\prime}$ is quasiconformal in a region containing $\mathrm{Cl} D^{\prime}$.

Proof: Suppose first that $f$ is quasiconformal in a neighborhood of every point of $\mathrm{Bd} D^{\prime}$. Then $f$ is quasiconformal in a region containing $\mathrm{Bd} D^{\prime}$ and we can use quasiconformal continuation to obtain $f^{\prime}$ satisfying (i)-(iv) ([15], II. 8.1).

In other cases choose $z_{0} \in \operatorname{Bd} D^{\prime}$. Let $h: D^{\prime} \rightarrow H$ and $u: f\left(D^{\prime}\right) \rightarrow H$ be conformal mappings such that $h\left(z_{0}\right)=\infty=u\left(f\left(z_{0}\right)\right)$. Define $w: H \rightarrow H$ by (1.6) with the boundary values $\varphi=u \circ f \circ h^{-1}: R$ and set

$$
f^{\prime}=\left\{\begin{array}{l}
u^{-1} \circ w \circ h \text { in } D^{\prime}, \\
f \text { elsewhere in } D .
\end{array}\right.
$$

Then (i) and (ii) hold. If $f$ is quasiconformal in a neighborhood of $z \in \operatorname{Bd} D^{\prime}$, then the mapping $\varphi$ is quasisymmetric on an interval containing $h(z)$ as proved in 6 , and (iii) follows.

\section{§ 2. Homotopic mappings of Riemann surfaces}

1. Let $S=D / G$ and $S^{\prime}=D / G^{\prime}$ be Riemann surfaces, $(D, \pi)$ and 
$\left(D, \pi^{\prime}\right)$ their universal covering surfaces, and $f: S \rightarrow S^{\prime}$ a continuous mapping. As a generalization of the path lifting, we construct a lifting $\tilde{f}: D \rightarrow D$ of $f$ as follows: Choose $p \in S, z \in \pi^{-1}(p)$ and $z^{\prime} \in\left(\pi^{\prime}\right)^{-1}\left(p^{\prime}\right), p^{\prime}$ $=f(p)$. Let $\zeta \in D$ and let $\tilde{\gamma}: I \rightarrow D$ be a path from $z$ to $\zeta$. Define $\tilde{f}(\zeta)$ as the end point of the lifting of $f \circ \pi \circ \tilde{\gamma}$ from $z^{\prime}$. It follows that $\tilde{f}$ is well defined and continuous. Moreover, $\tilde{f}$ satisfies the following equation:

$$
f \circ \pi=\pi^{\prime} \circ \tilde{f}
$$

If $S^{\prime}$ is not simply connected, there exist different liftings of $f$ (i.e., continuous mappings $\tilde{f}: D \rightarrow D$ satisfying (2.1)). On the other hand, a connectedness argument shows that two liftings $\tilde{f}$ and $\tilde{f}^{\prime}$ of $f$ coincide if there is a point $z \in D$ such that $\tilde{f}(z)=\tilde{f}^{\prime}(z)$. Therefore, if $z^{\prime}$ runs through the set $\left(\pi^{\prime}\right)^{-1}\left(p^{\prime}\right)$, all liftings of $f$ are obtained by the above construction of $\tilde{f}$. Especially, this observation yields the following lemma:

Lemma 2.1. If $\tilde{f}$ and $\tilde{f}^{\prime}$ are liftings of $f$, there exists a unique covering transformation $g^{\prime} \in G^{\prime}$ such that $\tilde{f}^{\prime}=g^{\prime} \circ \tilde{f}$.

By Lemma 2.1, every lifting $\tilde{f}$ of $f$ induces a homomorphism $\tilde{f}_{*}$ : $G \rightarrow G^{\prime}$ satisfying

$$
\tilde{f}_{*}(g) \circ \tilde{f}=\tilde{f} \circ g .
$$

If $f$ is a homeomorphism, then $\tilde{f}_{*}$ is an isomorphism.

Note that $\tilde{f}$ inherits many properties from $f$. For instance, $\tilde{f}$ is homeomorphic, differentiable, quasiconformal or conformal simultaneously with $f$.

2. Let $\operatorname{Hom}\left(G, G^{\prime}\right)$ be the set of all homomorphisms between $G$ and $G^{\prime}$. We define in $\operatorname{Hom}\left(G, G^{\prime}\right)$ an equivalence relation $\sim$ by setting $j \sim j^{\prime}$ if there exists an inner automorphism $A: G^{\prime} \rightarrow G^{\prime}$ such that $j^{\prime}=A \circ j$. Let $[j]$ denote the equivalence class of $j$.

By Lemma 2.1, $f$ defines a unique equivalence class $\left[\tilde{f}_{*}\right]$ in Hom $\left(G, G^{\prime}\right)$. Moreover, if $j \in\left[\tilde{f}_{*}\right]$, then there is a lifting $\tilde{f}^{\prime}$ of $f$ such that $j=\tilde{f}_{*}^{\prime}$.

In fact, the class $\left[\tilde{f}_{*}\right]$ does not change if $f$ is deformed continuously:

Lemma 2.2. Continuous mappings $f_{i}: S \rightarrow S^{\prime}, i=0,1$, are homotopic if and only if $\left[\tilde{f}_{0 *}\right]=\left[\tilde{f}_{1 *}\right]$. 
For a proof, see [11] and [4]. Note that Lemma 2.2 can be stated also as follows: $f_{0}$ and $f_{1}$ are homotopic if and only if there are liftings $\tilde{f}_{0}$ and $\tilde{f}_{1}$ such that $\tilde{f}_{0 *}=\tilde{f}_{1 *}$.

3. Let $C\left(S, S^{\prime}\right)$ be the set of continuous mappings $f: S \rightarrow S^{\prime}$, and let $\simeq$ denote the equivalence relation homotopy defines in $C\left(S, S^{\prime}\right)$. By Lemma 2.2, we can define an injective mapping $F: C\left(S, S^{\prime}\right) / \simeq \rightarrow$ $\operatorname{Hom}\left(G, G^{\prime}\right) / \sim$ by $[f] \stackrel{F}{\mapsto}\left[\tilde{f}_{*}\right]$.

In the classical surface theory, $S$ and $S^{\prime}$ are supposed to be compact. Then, by a theorem of Hopf [11], $F$ is a bijection. Moreover, given an isomorphism $j: G \rightarrow G^{\prime}$, there exists a homeomorphism $f: S \rightarrow S^{\prime}$ such that $\tilde{f}_{*}=j$. This result is due to Nielsen [17] and is referred to as Nielsen's theorem in the following.

In the general case, Nielsen's theorem is trivially false: choose $S=\hat{C}$ and $S^{\prime}=C$. If $D$ is required to be the universal covering surface of $S$ and $S^{\prime}$, a counter example is obtained as follows: let $S$ be a disk punctured twice and $S^{\prime}$ a torus punctured once. Then $S$ and $S^{\prime}$ hare isomorphic fundamental groups (free groups generated by two elements), and thus $G$ and $G^{\prime}$ are isomorphic ([3] I $19 \mathrm{~A}$ ). On the other hand, $S$ and $S^{\prime}$ are not topologically equivalent because there are Jordan curves $\gamma: I \rightarrow S^{\prime}$ such that $S^{\prime} \backslash \gamma(I)$ is connected.

4. We conclude this section by presenting a new proof to the following well-known theorem (Teichmüller [18], [19] and Bers [4], [5]):

Theorem 2.1. Let $S$ and $S^{\prime}$ be compact Riemann surfaces. Then the homotopy class of a sense-preserving homeomorphism $f: S \rightarrow S^{\prime}$ contains quasiconformal mappings.

Proof: Suppose first that $D$ is the universal covering surface of $S$ and $S^{\prime}$. Then, by the compactness of $S$. there exists a fundamental set $A$ of $G$ contained in a disk $\{z|| z \mid<1-\varepsilon\}$ for some $\varepsilon, 0<\varepsilon<1$. Let $\left\{D_{1}, \ldots, D_{n}\right\}$ be an open covering of $A$ with disks such that $\pi \mid \mathrm{Cl} D_{k}$ is injective, $k=1, \ldots, n$, and let $\tilde{f}$ be a lifting of $f$. D wine $\tilde{f}_{0}=\tilde{f}$ and inductively $\tilde{f}_{k}, k=1, \ldots, n$, as follows:

$$
\tilde{f}_{k}= \begin{cases}\tilde{f}_{*}(g) \circ \tilde{f}_{k-1}^{\prime} \circ g^{-1} & \text { in } g\left(D_{k}\right) \text { for all } g \in G, \\ \tilde{f}_{k-1} & \text { elsewhere in } D,\end{cases}
$$

where the homeomorphism $\tilde{f}_{k-1}^{\prime}$ satisfies the conditions of Lemma 1.1 with respect to $\tilde{f}_{k-1}$ and $D_{k}$. One verifies by induction that $\tilde{f}_{k}$ is locally quasiconformal in the open set 


$$
\bigcup_{g \in G} \bigcup_{i=1}^{k} g\left(D_{i}\right) \text {. }
$$

Hence $\tilde{f}_{n}$ is a quasiconformal self-mapping of $D$.

On the other hand, $\tilde{f_{*}}(g) \circ \tilde{f_{k}}=\tilde{f_{k}} \circ g$ holds for all $k=0,1, \ldots, n$ and $g \in G$. Consequently there is a unique continuous mapping $f_{n}: S \rightarrow S^{\prime}$ such that $\tilde{f}_{n}$ is a lifting of $f_{n}$. By Lemma $2.2, f \simeq f_{n}$, and by the quasiconformality of $\tilde{f}_{n}$, the mapping $f_{n}$ is quasiconformal.

If, finally, $S$ and $S^{\prime}$ are tori, we can apply the above proof replacing $D$ by $C$.

The preceding proof is modified from one Bers gives in [5]. It can be interpreted also as follows: Cover $S$ with finitely many parametric disks $U_{1}, \ldots, U_{n}$, i.e., the images of the disks $D_{k}$ under the projection mapping $\pi$. Set $f_{0}=f$ and define inductively $f_{k}=f_{k-1}$ in $S \backslash U_{k}$ and $f_{k} \mid U_{k}$ to be the locally quasiconformal mapping with the boundary values $\varphi=f_{k-1} \mid \operatorname{Bd} U_{k}$ constructed in Lemma 1.1. Then $f_{n}$ is a quasiconformal mapping homotopic to $f$.

Slightly modifying the above proof we obtain the following result: If $S$ is an arbitrary Riemann surface, $f: S \rightarrow S^{\prime}$ a sense-preserving homeomorphism, and $U \subset S$ a region with a compact closure, then the homotopy class of $f$ contains homeomorphisms quasiconformal in $U$.

Theorem 2.1 does not hold for arbitrary Riemann surfaces, since e.g. $C$ and $D$ are topologically but not quasiconformally equivalent. An example constructed in 4.8 shows that Theorem 2.1 does not hold even if $D$ is the universal covering surface of $S$ and $S^{\prime}$.

\section{§ 3. The boundary mapping associated with an isomorphism}

1. We begin this section by summarizing some properties of the covering groups.

Let $G$ be a covering group acting in a disk or a half-plane $D^{\prime}$. If $g \in G$ is a hyperbolic transformation, the axis of $g$ is defined to be the circle or the straight line through the fixed points $P(g)$ and $N(g)$ orthogonal to $\mathrm{Bd} D^{\prime}$. If $g$ is parabolic, the point $P(g)=N(g)$ is called the axis of $g$. We denote by $A x(g)$ the axis of $g \in G \backslash\{i d\}$.

The set of the fixed points of all non-identity transformations of $G$ is denoted by $F i x(G)$. If $F i x(G)$ is dense in $\operatorname{Bd~} D^{\prime}$, the group $G$ is said to be of the first kind.

Let $G$ and $G^{\prime}$ be covering groups. We say that an isomorphism $j: G \rightarrow G^{\prime}$ is induced by a continuous mapping $f: A \rightarrow \hat{C}$ in a set $A \subset \hat{C}$ if the following conditions hold: 
(i) $g(A)=A$ for all $g \in G$,

(ii) $f \circ g=j(g) \circ f$.

2. In the following lemma, we list properties of covering groups.

Lemma 3.1. A covering group $G$ of $D^{\prime}$ has the following properties:

(i) If $z \in F i x(G)$, then the set $\{g \in G \mid g(z)=z\}$ is a maximal cyclic subgroup of $G$.

(ii) Let $h_{1}$ and $h_{2}$ be non-identity transformations of $G$ such that $N\left(h_{1}\right) \neq P\left(h_{2}\right)$, and $g_{n}=h_{2}^{n} \circ h_{1}^{n}$. Then $N\left(g_{n}\right) \rightarrow N\left(h_{1}\right)$ and $P\left(g_{n}\right) \rightarrow$ $P\left(h_{2}\right)$ as $n \rightarrow \infty$.

(iii) If $G$ is of the first kind and $A_{1}$ and $A_{2}$ are disjoint open subsets of $\mathrm{Bd} D^{\prime}$, then there is a $g \in G$ such that $P(g) \in A_{1}$ and $N(g) \in A_{2}$.

The first assertion is proved in [13] $\mathrm{I} 2 \mathrm{H}$, (ii) in [20] 1.4, and (iii) follows from (ii).

3. We next introduce a method to construct special covering groups.

Let $g$ be a hyperbolic or a parabolic transformation fixing the unit disk $D$. The isometric circle $I(g)$ of $g$ is defined by

$$
I(g)=\left\{z||(g)^{\prime}(z) \mid=1\right\}
$$

$(g)^{\prime}$ denoting the derivative of $g$. As proved e.g. in [10], $I(g)$ is a circle orthogonal to $K$ and to $A x(g), g(I(g))=I\left(g^{-1}\right)$, and $I(g)$ and $I\left(g^{-1}\right)$ have the same radius. If $g$ is parabolic, then $I(g)$ and $I\left(g^{-1}\right)$ are tangent to each other at $P(g)$, otherwise $I(g) \cap I\left(g^{-1}\right)=\varnothing$. The inside of $I(g)$ is mapped by $g$ onto the outside of $I\left(g^{-1}\right)$, and we have $\left|(g)^{\prime}(z)\right|>1$ inside $I(g)$ and $\left|(g)^{\prime}(z)\right|<1$ outside $I(g)$.

Let $F(g)$ denote the part of $D$ outside both $I(g)$ and $I\left(g^{-1}\right)$, i.e.,

$$
F(g)=\left\{z \in D \|(g)^{\prime}(z):<1 \&:\left(g^{-1}\right)^{\prime}(z),<1\right\} .
$$

For the identity transformation we define $F(i d)=D$. The region $F(g)$ is a fundamental domain of the group generated by $g$. Moreover, if $G$ is a covering group of $D$, then

$$
F(G)=\bigcap_{g \in G} F(g)
$$

is a fundamental domain of $G$. The boundary of $F(G)$ contains no arcs of $K$ if and only if $G$ is of the first kind.

We call a set $\left\{g_{1}, g_{2}, \ldots\right\}$ consisting of parabolic or hyperbolic transformations fixing $D$ a free combination if $D \backslash \mathrm{Cl} F\left(g_{i}\right) \subset F\left(g_{j}\right)$ whenever $i \neq j$. 
Lemma 3.2. Let $G$ be the group generated by a free combination $\left\{g_{1}, g_{2}, \ldots\right\}$. Then

( i) $G$ is a covering group of $D$,

(ii) $G$ is free in the generators $g_{1}, g_{2}, \ldots$,

(iii) $F(G)=\bigcap_{i} F\left(g_{i}\right)$.

Lemma 3.2 is proved in [10], section 25. It also follows from Theorem 2.6 in $[20]$.

4. Let $G$ and $G^{\prime}$ be covering groups of the first kind acting in $D^{\prime}$ and $D^{\prime \prime}$, respectively. A homeomorphism $\varphi: \operatorname{Bd} D^{\prime} \rightarrow \operatorname{Bd} D^{\prime \prime}$ is called the boundary mapping of an isomorphism $j: G \rightarrow G^{\prime}$ if

$$
\varphi(P(g))=P(j(g))
$$

holds for all $g \in G \backslash\{i d\}$. Since $F i x(G)$ and $F i x\left(G^{\prime}\right)$ are dense in $\operatorname{Bd} D^{\prime}$ and $\mathrm{Bd} D^{\prime \prime}$, respectively, (3.1) defines $\varphi$ uniquely. On the other hand, there are isomorphisms which do not have boundary mappings; an example is constructed in 3.10 .

The existence of the boundary mapping of an isomorphism $j: G \rightarrow G^{\prime}$ is, in the following sense, invariant under Möbius transformations: Let $h$ and $h^{\prime}$ be Möbius transformations, $G_{1}=h G h^{-1}, G_{1}^{\prime}=h^{\prime} G^{\prime}\left(h^{\prime}\right)^{-1}$ and $j_{1}: G_{1} \rightarrow G_{1}^{\prime}$ the isomorphism defined by

$$
j_{1}\left(g_{1}\right)=h^{\prime} \circ j\left(h^{-1} \circ g_{1} \circ h\right) \circ\left(h^{\prime}\right)^{-1} .
$$

Then the boundary mappings $\varphi$ and $\varphi_{1}$ of $j$ and $j_{1}$ exist simultaneously and

$$
\varphi_{1}=h^{\prime} \circ \varphi \circ h^{-1} \text {. }
$$

This possibility to transform the given covering group will be used repeatedly in the following. For instance, in the rest of this section we shall develop the general properties of the boundary mapping of an jsomorphism considering only covering groups acting in $D$.

5. By the following theorem, the boundary mapping $\varphi$ is the only homeomorphism, if any, which induces $j: G \rightarrow G^{\prime}$ in the closure of $F i x(G)$.

Theorem 3.1. Let $G$ and $G^{\prime}$ be covering groups of the first kind acting in $D$ and $j: G \rightarrow G^{\prime}$ an isomorphism.

(i) If the boundary mapping $\varphi$ of $j$ exists, then $\varphi$ induces $j$ in $K$.

(ii) If $\varphi: K \rightarrow \hat{C}$ is a continuous injection inducing $j$ in $K$, then $\varphi$ is the boundary mapping of $j$.

(iii) If $f: D \cup K \rightarrow D \cup K$ is a homeomorphism such that $f \mid D$ induces $j$ in $D$, then $f \mid K$ is the boundary mapping of $j$. 
Proof: To prove (i), let $g$ and $h$ be non-identity transformations of $G$. Then $(\varphi \circ g)(P(h))=\varphi\left(P\left(g \circ h \circ g^{-1}\right)=P\left(j\left(g \circ h \circ g^{-1}\right)\right)=P\left(j(g) \circ j(h) \circ j(g)^{-1}\right)\right.$ $=j(g)(P(j(h)))=(j(g) \circ \varphi)(P(h))$. Therefore

$$
\varphi \circ g|F i x(G)=j(g) \circ \varphi| F i x(G) .
$$

Because $\varphi$ is continuous, (i) holds.

In (ii), choose $g \in G \backslash\{i d\}$ and $z \in K \backslash\{N(g)\}$ such that $\varphi(z) \neq$ $N(j(g))$. Then $\varphi\left(g^{n}(z)\right)=j(g)^{n}(\varphi(z))$ and letting $n \rightarrow \infty, \varphi(P(g))=$ $P(j(g))$ follows.

By continuity, $f \mid K$ induces $j$ in $K$. Thus (iii) follows from (ii).

Suppose that $D / G$ and $D / G^{\prime}$ are compact. Applying (iii), the existence of the boundary mapping of an isomorphism $j: G \rightarrow G^{\prime}$ then follows from Nielsen's theorem and Theorem 2.1.

In (iii), $f$ has a natural extension to $\hat{C}$. If we define

$$
f^{*}(z)=\left\{\begin{array}{lll}
f(z) & \text { if } & |z| \leq 1 \\
f\left(z^{*}\right)^{*} & \text { if } & |z|>1
\end{array}\right.
$$

where $z^{*}=1 / \bar{z}$, then $f^{*}: \hat{C} \rightarrow \hat{C}$ is a homeomorphism inducing $j$ in $\hat{C}$

6. The rest of this section deals with different characterizations for the existence of a boundary mapping. In the following theorem, we summarize results recently published by Tukia [20].

Theorem 3.2. Let $G$ and $G^{\prime}$ be covering groups of the first kind acting in $D$ and $j: G \rightarrow G^{\prime}$ an isomorphism. Then the following conditions are equivalent:

(i) The boundary mapping $\varphi$ of $j$ exists.

(ii) If $g_{1}$ and $g_{2}$ are non-identity transformations of $G$, then $A x\left(g_{1}\right) \cap A x\left(g_{2}\right) \neq \varnothing$ if and only if $A x\left(j\left(g_{1}\right)\right) \cap A x\left(j\left(g_{2}\right)\right) \neq \varnothing$.

(iii) There is a homeomorphism $f: D \rightarrow D$ inducing $j$ in $D$.

(iv) There is a homeomorphism $f^{*}: \hat{C} \rightarrow \hat{C}$ inducing $j$ in $\hat{C}$.

The equivalence of (ii) and (iii) is proved by Tukia [20] (Lemma 3.4 and Theorem 3.6); see also Fenchel and Nielsen [9] and Marden [16].

By Corollary 3.5.1 in [20], every homeomorphism $f: D \rightarrow D$ inducing $j$ in $D$ admits a homeomorphic extension to $K$. Consequently (iii) implies (iv) by formula (3.3). On the other hand, if $f^{*}$ in (iv) maps $D$ onto $\{z|| z \mid>1\}$, then $1 / \bar{f}^{*}$ maps $D$ onto itself and induces $j$ in $D$. Thus (iv) implies (iii).

By Proposition 3.5 in [20], (ii) implies (j). Conversely, (i) implies (ii) by formula (3.1). 
7. Let us make some complementary remarks to Theorem 3.2. Retaining the assumptions on $G$ and $G^{\prime}$, suppose that an isomorphism $j: G \rightarrow G^{\prime}$ has the following property:

(A) $j(g)$ is parabolic if and only if $g$ is parabolic.

Then it follows from (i) in Lemma 3.1 that there is a bijection $\Phi: F i x(G)$ $\rightarrow$ Fix $\left(G^{\prime}\right)$ defined by

$$
\Phi(P(g))=P(j(g)), g \in G \backslash\{i d\} .
$$

In order to extend $\Phi$ to the boundary mapping $\varphi$ of $j$, we consider the following condition:

(B) If $z_{1}, \ldots, z_{4}$ are distinct points in $F i x(G)$, then $\left(z_{1}, z_{2}, z_{3}, z_{4}\right)$ $>1$ if and only if $\left(\Phi\left(z_{1}\right), \Phi\left(z_{2}\right), \Phi\left(z_{3}\right), \Phi\left(z_{4}\right)\right)>1$.

Geometrically, $\left(z_{1}, z_{2}, z_{3}, z_{4}\right)>1$ if and only if $z_{2}$ and $z_{4}$ lie in different components of $K \backslash\left\{z_{1}, z_{3}\right\}$, i.e., $z_{1}$ and $z_{3}$ separate $z_{2}$ and $z_{4}$.

We call $g \in G \backslash\{i d\}$ a boundary transformation if it has the following property: $A x(g) \cap A x(h) \neq \varnothing$ for an $h \in G \backslash\{i d\}$ only if $A x(g)=A x(h)$. (This definition agrees with the corresponding one given by Marden in [16].) By Lemma 3.1, every parabolic transformation $g \in G$ is a boundary transformation, and if $G$ is of the first kind, all boundary transformations are parabolic.

Theorem 3.3. Let $G$ and $G^{\prime}$ be covering groups of the first kind acting in $D$ and $j: G \rightarrow G^{\prime}$ an isomorphism. Then the boundary mapping $\varphi$ of $j$ exists if at least one of the following conditions is satisfied:

(i) $D / G$ is compact,

(ii) $G$ is finitely generated and (A) holds,

(iii) (A) and (B) hold.

Conversely, if $\varphi$ exists, then (A) and (B) hold.

Proof: The sufficiency of (i) is already stated in 3.5. Secondly, suppose that (ii) holds. Then $j(g)$ is a boundary transformation if and only if $g$ is one, and it follows from a theorem of Fenchel-Nielsen [9] and Marden [16] that $j$ is induced by a homeomorphism in $D$. Thus by Theorem 3.2, $\varphi$ of $j$ exists.

The necessity of the conditions (A) and (B) is clear. Conversely, suppose that (A) holds. Then $\Phi$ defined by (3.4) exists, and since Fix $(G)$ and $F i x\left(G^{\prime}\right)$ are dense in $K$, the existence of $\varphi$ follows from (B) by standard topological arguments. For the sake of completeness, we repeat the construction of $\varphi$. 
Let $z \in K$ and $\left\{z_{n}\right\} \subset F i x(G)$ such that $z_{n} \rightarrow z$ as $n \rightarrow \infty$. We show that there is a $w \in K$ such that $\Phi\left(z_{n}\right) \rightarrow w$. If not, then there are two different points $w_{1}$ and $w_{2}$ and two subsequences $\left\{z_{1 k}\right\}$ and $\left\{z_{2 k}\right\}$ of $\left\{z_{n}\right\}$ such that $\Phi\left(z_{i k}\right) \rightarrow w_{i}$ as $k \rightarrow \infty, i=1,2$. Since $G^{\prime}$ is of the first kind and $\Phi$ is a bijection, we can choose $\zeta_{1}$ and $\zeta_{2}$ in Fix $(G)$ and a $k_{0}>0$ such that $\zeta_{1} \neq z \neq \zeta_{2}$ and $\left(\Phi\left(\zeta_{1}\right), \Phi\left(z_{1 k}\right), \Phi\left(\zeta_{2}\right), \Phi\left(z_{2 k}\right)\right)>1$ for all $k>k_{0}$. On the other hand, since $z_{i k} \rightarrow z \neq \zeta_{i}, i=1,2, \zeta_{1}$ and $\zeta_{2}$ cannot separate the points $z_{1 k}$ and $z_{2 k}$ from some $k=k_{1}$ onwards. This contradicts (B). It follows similarly that $\Phi\left(z_{n}^{\prime}\right) \rightarrow w$ whenever $z_{n}^{\prime} \rightarrow z$ and $\left\{z_{n}^{\prime}\right\} \subset F i x(G)$.

Define $\varphi: K \rightarrow K$ by $\varphi(z)=w$. Then (3.1) automatically holds. The above construction of $\varphi$ is symmetric with respect to $j$ and $j^{-1}$. Therefore, if $\Phi\left(z_{n}\right) \rightarrow w$ as $n \rightarrow \infty$, then we have a unique $z \in K$ such that $z_{n} \rightarrow z$. This proves the bijectivity of $\varphi$. To prove that $\varphi$ and $\varphi^{-1}$ are continuous, we show that for any points $z_{1}, \ldots, z_{4} \in K$ we have $\left(\varphi\left(z_{1}\right), \varphi\left(z_{2}\right), \varphi\left(z_{3}\right), \varphi\left(z_{4}\right)\right)>1$ if and only if $\left(z_{1}, z_{2}, z_{3}, z_{4}\right)>1$. Suppose that $\left(z_{1}, z_{2}, z_{3}, z_{4}\right)>1$, and let $z_{\text {in }} \in F i x(G)$ be points such that $z_{\text {in }} \rightarrow z_{i}$ as $n \rightarrow \infty, i=1,2,3,4$. Then by $(\mathrm{B}),\left(\Phi\left(z_{1 n}\right), \Phi\left(z_{2 n}\right), \Phi\left(z_{3 n}\right), \Phi\left(z_{4 n}\right)\right)$ $>1$ from some $n=n_{0}$ onwards. Since $\Phi\left(z_{i n}\right) \rightarrow \varphi\left(z_{i}\right)$ as $n \rightarrow \infty$, $\left(\varphi\left(z_{1}\right), \varphi\left(z_{2}\right), \varphi\left(z_{3}\right), \varphi\left(z_{4}\right)\right)>1$ follows. Similarly, $\left(z_{1}, z_{2}, z_{3}, z_{4}\right)>1$ if $\left(\varphi\left(z_{1}\right), \varphi\left(z_{2}\right), \varphi\left(z_{3}\right), \varphi\left(z_{4}\right)\right)>1$. Therefore, if $z \in K$ and $\left\{z_{n}\right\} \subset K$ such that $z_{n} \rightarrow z$ as $n \rightarrow \infty$, it follows as above that $\varphi\left(z_{n}\right) \rightarrow \varphi(z)$. Thus $\varphi$ is continuous. The continuity of $\varphi^{-1}$ can be proved similarly.

Since a covering group $G$ corresponding to a compact Riemann surface $D / G$ contains no parabolic elements (see [4]), the condition (i) is a special case of (ii).

8. From Theorems 3.2 and 3.3 it follows that the axis condition (ii) in Theorem 3.2 holds if and only if (A) and (B) are valid. This can also be proved directly as follows:

It is clear that (A) and (B) together imply (ii). It follows from Lemma 3.1 that (ii) implies (A). Hence, it is sufficient to show that (A) and (ii) together imply $(\mathrm{B})$.

Let $z_{1}, \ldots, z_{4}$ be points in Fix $(G)$ with $\left(z_{1}, z_{2}, z_{3}, z_{4}\right)>1$. Choose $h_{i} \in G$ such that $z_{1}=N\left(h_{1}\right), z_{2}=N\left(h_{2}\right), z_{3}=P\left(h_{3}\right), z_{4}=P\left(h_{4}\right)$, and set $g_{1 n}=h_{3}^{n} \circ h_{1}^{n}$ and $g_{2 n}=h_{4}^{n} \circ h_{2}^{n}$. Then, by (ii) in Lemma $3.1, N\left(g_{\text {in }}\right.$ ) $\rightarrow z_{i}$ and $P\left(g_{i n}\right) \rightarrow z_{i+2}$ as $n \rightarrow \infty, i=1,2$. Thus there is a $n_{0}>0$ such that $A x\left(g_{1 n}\right) \cap A x\left(g_{2 n}\right) \neq \varnothing$ for $n>n_{0}$. By (A), $\Phi$ defined by (3.4) exists, and $N\left(j\left(g_{i n}\right)\right) \rightarrow \Phi\left(z_{i}\right)$ and $P\left(j\left(g_{i n}\right)\right) \rightarrow \Phi\left(z_{i+2}\right)$ as $n \rightarrow \infty$, $i=1,2$. From $A x\left(j\left(g_{1 n}\right)\right) \cap A x\left(j\left(g_{2 n}\right)\right) \neq \varnothing, n>n_{0}$, it then follows that $\left(\Phi\left(z_{1}\right), \Phi\left(z_{2}\right), \Phi\left(z_{3}\right), \Phi\left(z_{4}\right)\right)>1$. Similarly, if $\left(\Phi\left(z_{1}\right), \Phi\left(z_{2}\right), \Phi\left(z_{3}\right)\right.$, $\left.\Phi\left(z_{4}\right)\right)>1$, then $\left(z_{1}, z_{2}, z_{3}, z_{4}\right)>1$. 
9. The following theorem tells more about the groups $G$ and $G^{\prime}$ if the boundary mapping does not exist.

Theorem 3.4. Let $G$ and $G^{\prime}$ be covering groups of the first kind acting in $D$ and $j: G \rightarrow G^{\prime}$ an isomorphism. The boundary mapping of $j$ does not exist if and only if there is a subgroup $G_{1}$ of $G$ such that one of the Riemann surfaces $D / G_{1}$ and $D_{i} j\left(G_{1}\right)$ is homeomorphic to a disk punctured twice and the other to a torus punctured once.

Proof: Suppose first that there exists a subgroup $G_{1} \subset G$ such that one of the Riemann surfaces $D / G_{1}$ and $D / j\left(G_{1}\right)$ is homeomorphic to a disk punctured twice and the other to a torus punctured once. Then $D / G_{1}$ and $D / j\left(G_{1}\right)$ are not topologically equivalent. Therefore $j$ cannot be induced by a homeomorphism in $D$. By Theorem 3.2, the boundary mapping of $j$ does not exist.

Suppose that the boundary mapping of $j$ does not exist. Then the axis condition (ii) in Theorem 3.2 does not hold. Since we can replace $j$ by $j^{-1}$, we may assume that there are hyperbolic transformations $g_{1}$ and $g_{2}$ in $G$ such that $A x\left(g_{1}\right) \cap A x\left(g_{2}\right) \neq \varnothing$ and $A x\left(j\left(g_{1}\right)\right) \cap A x\left(j\left(g_{2}\right)\right)$ $=\varnothing$. Moreover, we may assume that $\left\{g_{1}, g_{2}\right\}$ and $\left\{j\left(g_{1}\right), j\left(g_{2}\right)\right\}$ are free combinations. (By (i) in Lemma 3.1, this is achieved by replacing $g_{1}$ and $g_{2}$ by $g_{1}^{m}$ and $g_{2}^{n}$, where $m$ and $n$ are sufficiently large.) Let $G_{1}$ be the group generated by $\left\{g_{1}, g_{2}\right\}$. By (iii) in Lemma 3.2, we know the fundamental domains $F\left(G_{1}\right)$ and $F\left(j\left(G_{1}\right)\right)$, and it follows that $D / G_{1}$ is homeomorphic to a torus punctured once and $D / j\left(G_{1}\right)$ to a disk punctured twice.

10. We conclude this section with an example of an isomorphism whose boundary mapping does not exist.

Define four Möbius transformations $g_{1}, g_{2}, g_{1}^{\prime}, g_{2}^{\prime}$ fixing $D$ by the following requirements:

$$
\begin{aligned}
& I\left(g_{1}\right)=I\left(g_{1}^{\prime}\right)=\{z|| z-(1+i) \mid=1\}, \\
& I\left(g_{1}^{-1}\right)=I\left(g_{2}^{\prime}\right)=\{z|| z-(-1+i) \mid=1\}, \\
& I\left(g_{2}\right)=I\left(\left(g_{1}^{\prime}\right)^{-1}\right)=\{z|| z-(-1-i) \mid=1\}, \\
& I\left(g_{2}^{-1}\right)=I\left(\left(g_{2}^{\prime}\right)^{-1}\right)=\{z|| z-(1-i) \mid=1\} .
\end{aligned}
$$

Then $g_{1}$ and $g_{2}$ are parabolic whereas $g_{1}^{\prime}$ and $g_{2}^{\prime}$ are hyperbolic. Let $G$ and $G^{\prime}$ be the groups generated by $\left\{g_{1}, g_{2}\right\}$ and $\left\{g_{1}^{\prime}, g_{2}^{\prime}\right\}$, respectively.

Since $\left\{g_{1}, g_{2}\right\}$ and $\left\{g_{1}^{\prime}, g_{2}^{\prime}\right\}$ evidently are free combinations, $G$ and $G^{\prime}$ are covering groups by (i) in Lemma 3.2. Since the boundary of $F(G)$ $=F\left(G^{\prime}\right)$ contains no arcs of $K$, the groups $G$ and $G^{\prime}$ are of the first kind. By (ii) in Lemma 3.2, we can define an isomorphism $j: G \rightarrow G^{\prime}$ 
by $j\left(g_{i}\right)=g_{i}^{\prime}, i=1,2$. Since (A) does not hold, $j$ has no boundary mapping.

Considering the fundamental domains of $G$ and $G^{\prime}$, we see that $D / G$ is homeomorphic to a disk punctured twice and $D / G^{\prime}$ to a torus punctured once. Thus by Theorem 3.4, the example constructed is the simplest possible.

\section{§ 4. The dilatation of an isomorphism}

1. In this section we define a measure $\delta(j)$ for the distortion of an isomorphism $j: G \rightarrow G^{\prime}$. We shall see that $\delta(j)$ has some formal analogy with the maximal dilatation of a homeomorphism.

Let $G$ and $G^{\prime}$ be covering groups of the first kind and $j: G \rightarrow G^{\prime}$ an isomorphism. If $x(g)$ is the multiplier of $g \in G$ (see 1.4), let $A(j)$ be the set of real numbers $a \geq 1$ for which

$$
\varkappa(g)^{1 / a} \leq \varkappa(j(g)) \leq \varkappa(g)^{a}
$$

holds for all $g \in G$. If $\left\{a_{n}\right\} \subset A(j)$ such that $a_{n} \rightarrow a_{0}$ as $n \rightarrow \infty$ and if $g_{0} \in G$ is fixed, then (4.1) holds for $g_{0}$ and all numbers $a_{n}$. Therefore (4.1) is valid also for $g_{0}$ and $a_{0}$, and it follows that $a_{0} \in A(j)$, i.e., $A(j)$ is a closed set. We call $\delta(j)=\min a, a \in A(j)$, the dilatation of $j$. (Thus $\delta(j)=\infty$ if and only if $A(j)=\varnothing$.) Note that the condition (A) in 3.7 holds whenever $A(j) \neq \varnothing$.

Let $j^{\prime}: G^{\prime} \rightarrow G^{\prime \prime}$ be another isomorphism with a finite dilatation. Then it follows from (4.1) that

$$
\delta\left(j^{\prime} \circ j\right) \leq \delta\left(j^{\prime}\right) \delta(j) .
$$

From (1.2) we see that $\delta(j)=1$ if $j$ is induced by a Möbius transformation. Thus by $(4.2), \delta(j)$ is invariant under Möbius transformations: if $j_{1}$ is defined by $(3.2)$, then $\delta\left(j_{1}\right)=\delta(j)$. Also analogously with the maximal dilatation of a homeomorphism, it follows from (4.1) that $\delta(j)=\delta\left(j^{-1}\right)$.

2. By the following theorem, the dilatation of an isomorphism induced by a quasiconformal mapping is finite.

Theorem 4.1. Let $G$ and $G^{\prime}$ be covering groups of the first kind acting in $D^{\prime}$ and $D^{\prime \prime}$, respectively. If an isomorphism $j: G \rightarrow G^{\prime}$ is induced by a quasiconformal mapping $f: D^{\prime} \rightarrow D^{\prime \prime}$, then $\delta(j) \leq K(f)$, where $K(f)$ is the maximal dilatation of $f$. 
Proof: By (iii) in Theorem 3.1 (or by Theorem 3.2), the boundary mapping of $j$ exists. Therefore (A) in 3.7 holds, i.e., $x(j(g))=1$ if and only if $x(g)=1$.

Let $g \in G$ be hyperbolic. By (3.3) we may assume that $f$ is defined in the whole plane $\hat{C}$, and by the invariance of $K(f)$ and $\delta(j)$ under a Möbius transformation, we may assume that $P(g)=P(j(g))=\infty$ and $N(g)=N(j(g))=0$.

Set $g^{\prime}=j(g)$, and $k=\varkappa(g), k^{\prime}=\varkappa\left(g^{\prime}\right)$. Then $g(z)=k z$ and $g^{\prime}(z)$ $=k^{\prime} z$, and since $f$ induces $j$, we have

$$
f\left(k^{n} z\right)=\left(k^{\prime}\right)^{n} f(z)
$$

for $n=0, \pm 1, \pm 2, \ldots$. Let $B_{n}$ be the annulus bounded by the circles $|z|=1$ and $|z|=k^{n}, n=1,2, \ldots$. We approximate the ring domain $B_{n}^{\prime}=f\left(B_{n}\right)$ by an annulus $B_{n}^{\prime \prime}$ as follows: Let $\xi_{1}=\min _{\vartheta}\left|f\left(e^{i \vartheta}\right)\right|$ and $\xi_{2}=\max \left|f\left(e^{i \vartheta}\right)\right|$. Then $\xi_{1}>0$ and $\xi_{2}<\infty$. and we set

$$
B_{n}^{\prime \prime}=\left\{z: \xi_{1}<|z|<\left(k^{\prime}\right)^{n} \xi_{2}\right\} .
$$

It follows from (4.3) that $B_{n}^{\prime} \subset B_{n}^{\prime \prime}$. Moreover, $B_{n}^{\prime}$ separates the components of the complement of $B_{n}^{\prime \prime}$. Thus ([15], I.6.6)

$$
M\left(B_{n}^{\prime}\right) \leq M\left(B_{n}^{\prime \prime}\right)=\log \frac{\left(k^{\prime}\right)^{n} \xi_{2}}{\xi_{1}}=n \log k^{\prime}+\log \left(\xi_{2} / \xi_{1}\right) .
$$

On the other hand, since $f$ is $K(f)$-quasiconformal

$$
M\left(B_{n}^{\prime}\right) \geq M\left(B_{n}\right) / K(f)=(n \log k) / K(f) .
$$

So we have

$$
\log k \leq K(f) \log k^{\prime}+(K(f) / n) \log \left(\xi_{2} / \xi_{1}\right),
$$

and letting $n \rightarrow \infty$, we conclude that $\log k \leq K(f) \log k^{\prime}$. Similarly, $\log k^{\prime} \leq K(f) \log k$. Thus $K(f) \in A(j)$. $\square$

If we assume that $h$ and $h^{\prime}$ in $(3.2)$ are quasiconformal mappings such that $G_{1}$ and $G_{1}^{\prime}$ are covering groups, then it follows that $\delta(j) /$ $\left(K(h) K\left(h^{\prime}\right)\right) \leq \delta\left(j_{1}\right) \leq \delta(j) K(h) K\left(h^{\prime}\right)$. In this sense, the dilatation of an isomorphism is quasi-invariant under quasiconformal mappings.

3. Theorem 4.1 combined with Nielsen's theorem and Theorem 2.1 implies that $\delta(j)<\infty$ whenever $D^{\prime} / G$ is compact. More generally, applying the theorem of Fenchel and Nielsen [9] referred to in Theorem 3.3 we obtain the following corollary for Theorem 4.1.

Corollary. Suppose that $G$ is finitely generated. Then $\delta(j)<\infty$ if and only if the condition (A) in 3.7 holds. 
Proof: If $\delta(j)<\infty$, then (A) holds. If, conversely, (A) holds, then by Theorem 3.3, the isomorphism $j$ is induced by a homeomorphism $f: D^{\prime} \rightarrow D^{\prime \prime}$. Representing $D^{\prime} / G$ and $D^{\prime \prime} / G^{\prime}$ as compact Riemann surfaces with finitely many punctures (see [2]) and applying Theorem 2.1 we see that there are quasiconformal mappings inducing $j$ in $D^{\prime}$ (for details, see [7] p. 71). Then by Theorem 4.1, $\delta(j)<\infty$.

4. Suppose that $j: G \rightarrow G^{\prime}$ is an isomorphism whose boundary mapping $\varphi$ exists. By the following theorem, $\delta(j)<\infty$ if there is a quasiconformal mapping $f: \hat{C} \rightarrow \hat{C}$ such that $f=\varphi$ in the closure of Fix $(G)$.

Theorem 4.2. Let $G$ and $G^{\prime}$ be covering groups of the first kind acting in the upper half plane $H$. Suppose that the boundary mapping $\varphi$ of an isomorphism $j: G \rightarrow G^{\prime}$ exists and $\varphi(\infty)=\infty$. If $\varphi$ is $\lambda$-quasisymmetric, then

$$
\delta(j) \leq \log 2 / \log (1+1 / \lambda)
$$

Proof: The quasisymmetry of an increasing homeomorphism $\varphi: \hat{R} \rightarrow \hat{R}$ is defined in 1.5. As in the proof of Theorem 4.1, it suffices to consider hyperbolic transformations $g \in G$ only.

Suppose first that $\infty$ is not a fixed point of $g$. Since we can replace $g$ by $g^{-1}$, we may assume that $P(g)<N(g)$. Then there are Möbius transformations $h_{1}$ and $h_{2}$ fixing $\infty$ such that $h_{1}(P(g))=h_{2}(P(j(g)))=0$ and $h_{1}(N(g))=h_{2}(N(j(g)))=1$. Since $h_{1} \mid \hat{R}$ and $h_{2} \mid \hat{R}$ are 1-quasisymmetric, $h_{2} \circ \varphi \circ h_{1}^{-1} \mid \hat{R}$ is $\lambda$-quasisymmetric. Therefore, we may assume that $P(g)=P(j(g))=0$ and $N(g)=N(j(g))=1$.

Set $g^{\prime}=j(g), k=\varkappa(g)$ and $k^{\prime}=\varkappa\left(g^{\prime}\right)$. Then

$$
g(z)=\frac{z}{(1-k) z+k}, \quad g^{\prime}(z)=\frac{z}{\left(1-k^{\prime}\right) z+k^{\prime}},
$$

and

$$
g(1 / 2)=1 /(1+k) .
$$

By $\lambda$-quasisymmetry of $\varphi$, for each $m=1,2, \ldots$,

$$
1 /(1+\lambda)^{m} \leq \varphi\left(1 / 2^{m}\right) \leq \lambda^{m} /\left(1+\lambda_{.}\right)^{m}
$$

Let $p$ be the natural number for which

$$
1 / 2^{p} \leq 1 /(1+k)<1 / 2^{p-1}
$$

Then by (4.5)

$$
1 /(1+\lambda)^{p} \leq \varphi(1 /(1+k))=\varphi(g(1 / 2))<\lambda^{p-1} /(1+\lambda)^{p-1} .
$$


Set $m=1$ in (4.5). Then by (i) in Theorem 3.1

$$
1 /\left(1+\lambda k^{\prime}\right)=g^{\prime}(1 /(1+\lambda)) \leq \varphi(g(1 / 2)) \leq g^{\prime}(\lambda /(1+\lambda))=\lambda /\left(k^{\prime}+\lambda\right) .
$$

By (4.6), $p \geq \log (1+k) / \log 2>p-1$, and thus

$$
(1 /(1+\lambda))^{\log (2+2 k) / \log 2}<\lambda /\left(k^{\prime}+\lambda\right)
$$

and

$$
(\lambda /(1+\lambda))^{\log (1 / 2+k / 2) / \log 2}>1 /\left(1+k^{\prime} \lambda\right) .
$$

Since $y^{\log x}=x^{\log y}$ for $x, y>0$, we get

$$
\begin{gathered}
(1 / \lambda)((1+k) / 2)^{\log (1+1 / \lambda) / \log 2}-1 / \lambda<k^{\prime}< \\
\lambda(2+2 k)^{\log (1+\lambda) / \log 2}-\lambda .
\end{gathered}
$$

This double inequality holds for all hyperbolic transformations of $G$ not fixing $\infty$. Especially, we may replace $g$ by $g^{n}, n=1,2, \ldots$, and since $x\left(g^{n}\right)=k^{n}$,

$$
\begin{gathered}
k^{\prime}<\left[\lambda\left(2+2 k^{n}\right)^{\log (1+i) / \log 2}-\lambda\right]^{1 / n}< \\
\lambda^{1 / n}\left(2+2 k^{n}\right)^{\log (1+\lambda) /(n \log 2)},
\end{gathered}
$$

and letting $n \rightarrow \infty$,

$$
k^{\prime} \leq k^{\log (1+\lambda) / \log 2}
$$

On the other hand, from some $n=n_{0}$ on

$$
\begin{gathered}
k^{\prime}>\left[(1 / \lambda)\left(\left(1+k^{n}\right) / 2\right)^{\log (1+1 / \lambda) / \log 2}-1 / \lambda\right]^{1 / n}> \\
(1 /(2 \lambda))^{1 / n}\left(k^{n} / 2\right)^{\log (1+1 / i)((n \log 2)},
\end{gathered}
$$

and letting $n \rightarrow \infty$,

$$
k^{\prime} \geq k^{\log (1+1 \%) \log 2} .
$$

If $\infty$ is a fixed point of a hyperbolic transformation $g \in G$, we may assume that $P(g)=P\left(g^{\prime}\right)=0, N(g)=N\left(g^{\prime}\right)=\infty$ and $\varphi(1)=1$. Then $g(z)=z / k$ and $g^{\prime}(z)=z / k^{\prime}$, and we get as above the following double inequality:

$$
1 /(1+\lambda) k^{\log (1+1 / \lambda) / \log 2}<k^{\prime}<\lambda(2 k)^{\log (1+\lambda) / \log 2}
$$

Thus, replacing $g$ by $g^{n}$ and letting $n \rightarrow \infty,(4.7)$ and (4.8) are obtained also in this case. Consequently

$$
\max (\log (1+\lambda) / \log 2, \log 2 / \log (1+1 / \lambda)) \in A(j) .
$$

Finally, one verifies by calculation that $\log (1+1 / \lambda) \log (1+\lambda) \leq$ $(\log 2)^{2}$, i.e., $\log 2 / \log (1+1 / \lambda) \in A(j)$. 
Suppose that $\varphi$ is 1-quasisymmetric. Then there is a Möbius transformation $h$ fixing $H$ such that $h \mid \hat{R}=\varphi$. Since $j(g)\left|R=h \circ g \circ h^{-1}\right| R$, it follows that $h$ induces $j$ in $\hat{C}$. Consequently $\delta(j)=1$. On the other hand, setting $\lambda=1$ in Theorem 4.2. one obtains $\delta(j) \leq 1$. Thus the inequality (4.4) is sharp if $\lambda=1$.

It follows from (4.4) that $\delta(j) / \lambda$ stays bounded as $\lambda \rightarrow \infty$, i.e., $\delta(j)=O(\lambda)$.

5. We prove next a special case of Nielsen's theorem (see 2.3) which holds also for open surfaces.

Theorem 4.3. Let $G$ and $G^{\prime}$ be covering groups of the first kind. An isomorphism $j: G \rightarrow G^{\prime}$ is induced by a Möbius transformation in $\hat{C}$ if and only if $\delta(j)=1$.

Proof: If $j$ is induced by a Möbius transformation, then $\delta(j)=1$ by the formula (1.2).

Suppose that $\delta(j)=1$. Let $g_{1}$ and $g_{2}$ be two hyperbolic transformations of $G$ without common fixed points. Set

$$
\beta\left(g_{1}, g_{2}\right)=-\left(N\left(g_{1}\right), N\left(g_{2}\right), P\left(g_{1}\right), P\left(g_{2}\right)\right)+1 .
$$

Since $F i x(G)$ is contained in a circle or in a straight line, $\beta\left(g_{1}, g_{2}\right)$ is always real, and $\beta\left(g_{1}, g_{2}\right)<0$ if and only if $A x\left(g_{1}\right) \cap A x\left(g_{2}\right)=\varnothing$.

Choose $g_{1}$ and $g_{2}$ such that $\beta\left(g_{1}, g_{2}\right)<0$. By $\delta(j)=1$, (A) in 3.7 holds. Thus $\beta\left(j\left(g_{1}\right), j\left(g_{2}\right)\right)$ is defined, and we show that

$$
\beta\left(g_{1}, g_{2}\right)=\beta\left(j\left(g_{1}\right), j\left(g_{2}\right)\right) .
$$

To prove (4.9), set $g_{i}^{\prime}=j\left(g_{i}\right)$ and $k_{i}=\varkappa\left(g_{i}\right)=\varkappa\left(g_{i}^{\prime}\right), i=1$, 2 . Since the validity of (4.9) does not change if we replace $G^{\prime}$ and $G^{\prime}$ by conjugate groups $G_{1}$ and $G_{1}^{\prime}$ and $j$ by the isomorphism $j_{1}$ defined br (3.2), we may assume that $G$ and $G^{\prime}$ act in $H$ and that $N^{\prime}\left(g_{2}\right)=N^{\prime}\left(g_{2}^{\prime}\right)=0, P\left(g_{2}\right)$ $=P\left(g_{2}^{\prime}\right)=\infty$ and $P\left(g_{1}\right)=P\left(g_{1}^{\prime}\right)=1$. Then $x=\mathcal{N}^{\prime}\left(g_{1}\right)=\beta\left(g_{1}, g_{2}\right)$.

Since $\delta(j)=1$, it follows from (1.4) that

$$
\chi\left(g_{1} \circ g_{2}\right)=\chi\left(g_{1}^{\prime} \circ g_{2}^{\prime}\right) \text {. }
$$

On the other hand, we can determine all values $x^{\prime}=N\left(g_{1}^{\prime}\right)=\beta\left(g_{1}^{\prime}, g_{2}^{\prime}\right)$ for which (4.10) holds: Since

$$
\left(g_{1} \circ g_{2}\right)(z)=\frac{\left(k_{1}-x\right) k_{2} z-\left(k_{1}-1\right) x}{\left(k_{1}-1\right) k_{2} z-k_{1} x+1},
$$

we get 


$$
\chi\left(g_{1} \circ g_{2}\right)=\frac{k_{1}+k_{2}}{\sqrt{k_{1} k_{2}}}\left|\frac{a-x}{1-x}\right|,
$$

where

$$
a=a\left(g_{1}, g_{2}\right)=\frac{k_{1} k_{2}+1}{k_{1}+k_{2}}>1 .
$$

Similarly, we get an expression for $\chi\left(g_{1}^{\prime} \circ g_{2}^{\prime}\right)$ replacing $x$ in $(4.11)$ by $x^{\prime}$. Then, since $x<0,(4.10)$ yields

$$
\frac{a-x}{1-x}=\left|\frac{a-x^{\prime}}{1-x^{\prime}}\right|
$$

and hence

$$
x^{\prime}=\left\{\begin{array}{l}
x \\
\frac{a(2-x)-x}{a+1-2 x} .
\end{array}\right.
$$

If we replace $g_{1}$ and $g_{2}$ in (4.10) by $g_{1}^{m}$ and $g_{2}^{n}, m, n>1$, the number $x^{\prime}$ obtained by (4.12) does not change. On the other hand, $a\left(g_{1}, g_{2}\right)<$ $a\left(g_{1}^{m}, g_{2}^{n}\right)$. Consequently, since the function

$$
a \mapsto \frac{a(2-x)-x}{a+1-2 x}
$$

is increasing, $x^{\prime}=x$ is the only possible value for $x^{\prime}$. Thus (4.9) is proved.

Since $\delta\left(j^{-1}\right)=1$, it follows, similarly, that if we choose $g_{1}^{\prime}$ and $g_{2}^{\prime}$ such that $\beta\left(g_{1}^{\prime}, g_{2}^{\prime}\right)<0$, then $\beta\left(j^{-1}\left(g_{1}^{\prime}\right), j^{-1}\left(g_{2}^{\prime}\right)\right)<0$. Thus the axis condition (ii) in Theorem 3.2 holds and the boundary mapping $\varphi$ of $j$ exists. (We still assume that $G$ and $G^{\prime}$ act in $H$ and that $\varphi(\infty)=\infty$.)

Finally, it follows from (4.9) that $\varphi$ preserves cross ratios. To prove this, let $-\infty<x_{1}<x_{2}<x_{3}<x_{4}<\infty$. By (iii) in Lemma 3.1 we can construct two sequences $\left\{g_{1 n}\right\}$ and $\left\{g_{2 n}\right\}$ of transformations of $G$ such that $N\left(g_{i n}\right) \rightarrow x_{i}$ and $P\left(g_{i n}\right) \rightarrow x_{i+2}$ as $n \rightarrow \infty, i=1,2$. Then by the continuity of $\varphi$,

$$
\begin{gathered}
\left(x_{1}, x_{2}, x_{3}, x_{4}\right)=-\lim _{n \rightarrow \infty} \beta\left(g_{1 n}, g_{2 n}\right)+1= \\
-\lim _{n \rightarrow \infty} \beta\left(j\left(g_{1 n}\right), j\left(g_{2 n}\right)\right)+1=\left(\varphi\left(x_{1}\right), \varphi\left(x_{2}\right), \varphi\left(x_{3}\right), \varphi\left(x_{4}\right)\right) .
\end{gathered}
$$

Hence there is a Möbius transformation $h$ such that $\varphi=h \mid \hat{R}$, and, as remarked at the end of 4.4 , it follows that $h$ induces $j$ in $\hat{C}$.

6. The results of this section have some applications. 
Let $G_{0}$ be a fixed covering group of the first kind and $J\left(G_{0}\right)$ the set of all isomorphisms $j: G_{0} \rightarrow G$ with finite dilatation. (Note that $G$ is allowed to vary.) We define in $J\left(G_{0}\right)$ an equivalence relation by setting $j_{1} \sim j_{2}$ if $\delta\left(j_{2} \circ j_{1}^{-1}\right)=1$. Let $E\left(G_{0}\right)$ denote the set of equivalence classes:

$$
E\left(G_{0}\right)=J\left(G_{0}\right) / \sim
$$

and denote the equivalence class of $j$ by $[j]$.

In $E\left(G_{0}\right)$ we can define a natural metric:

Theorem 4.4. Set $d\left(\left[j_{1}\right],\left[j_{2}\right]\right)=\log \delta\left(j_{2} ; j_{1}^{-1}\right)$. Then $\left(E\left(G_{0}\right), d\right)$ is a metric space.

Proof: Let $j_{i} \sim j_{i}^{\prime}, i=1,2$. Since $j_{2}^{\prime} \circ\left(j_{1}^{\prime}\right)^{-1}=\left(j_{2}^{\prime} \circ j_{2}^{-1}\right) \circ\left(j_{2} \circ j_{1}^{-1}\right) \circ$ $\left(j_{1} \circ\left(j_{1}^{\prime}\right)^{-1}\right)$, it follows from $(4.2)$ that $\delta\left(j_{2}^{\prime} \circ\left(j_{1}^{\prime}\right)^{-1}\right) \leq \delta\left(j_{2}=j_{1}^{-1}\right)$, and similarly $\delta\left(j_{2} \circ j_{1}^{-1}\right) \leq \delta\left(j_{2}^{\prime} \circ\left(j_{1}^{\prime}\right)^{-1}\right)$. Thus $d$ is well defined.

By definition, $d \geq 0$ and $d\left(\left[j_{1}\right],\left[j_{2}\right]\right)=0$ if and only if $j_{1} \sim j_{2}$. Since $\delta(j)=\delta\left(j^{-1}\right)$ for all isomorphisms. $d\left(\left[j_{1}\right],\left[j_{2}\right]\right)=d\left(\left[j_{2}\right],\left[j_{1}\right]\right)$.

To prove the triangle inequality, let $j_{i} \in J\left(G_{0}\right), i=1,2,3$. Then $j_{3} \circ j_{1}^{-1}=\left(j_{3} \circ j_{2}^{-1}\right) \circ\left(j_{2} \circ j_{1}^{-1}\right)$ and by $(4.2), \delta\left(j_{3} \circ j_{1}^{-1}\right) \leq \delta\left(j_{3} \circ j_{2}^{-1}\right) \delta\left(j_{2} \circ j_{1}^{-1}\right) \cdot \square$

By Theorem 4.3, all isomorphisms in [id] are induced by Möbius transformations. Thus $d([j],[i d])=\log \delta(j)$ is a measure for the deviation of $j$ from isomorphisms induced by conformal mappings in $\hat{C}$.

7. We now restrict ourselves to covering groups acting in $D$. Let $G_{0}$ be of the first kind and let $J_{q}\left(G_{0}\right)$ be the set of isomorphisms $j: G_{0} \rightarrow G$ induced by quasiconformal self-mappings of $D$. Denote the set of quasiconformal mappings inducing $j$ by $B(j)$.

We define in $J_{q}\left(G_{0}\right)$ an equivalence relation $\approx$ by setting $j_{1} \approx j_{2}$ if $B\left(j_{\Omega} \circ j_{1}^{-1}\right)$ contains Möbius transformations. The set

$$
T\left(G_{0}\right)=J_{q}\left(G_{0}\right) / \approx
$$

is the Teichmüller space of $G_{0}$, and the Teichmiiller metric $\varrho$ in $T\left(G_{0}\right)$ is defined by

$$
\varrho\left(\left[j_{1}\right],\left[j_{2}\right]\right)=\log \inf \left\{K(f) \quad f \in B\left(j_{2}=j_{1}^{-1}\right)\right\} .
$$

(By Lemma 2.2, these definitions agree with the definition of the Teichmüller space of the Riemann surface $D / G_{0}$.)

By Theorem 4.1, $J_{q}\left(G_{0}\right) \subset J\left(G_{0}\right)$, and it follows from Theorem 4.3 that the relations $\approx$ and $\sim$ are the same in $J_{q}\left(G_{0}\right)$ :

$$
T\left(G_{0}\right)=J_{q}\left(G_{0}\right) / \sim .
$$

By Theorem 4.1, $d\left(\left[j_{1}\right],\left[j_{2}\right]\right) \leq \varrho\left(\left[j_{1}\right],\left[j_{2}\right]\right)$ holds for all $\left[j_{i}\right] \in T\left(G_{0}\right), i$ 
$=1,2$, but it remains us an open question whether these metrics are equivalent in $T\left(G_{0}\right)$.

8. To conclude this section, we construct two covering groups $G$ and $G^{\prime}$ and an isomorphism $j: G \rightarrow G^{\prime}$ satisfying the following conditions:

( i) $G$ and $G^{\prime}$ act in $D$ and are of the first kind,

(ii) $j: G \rightarrow G^{\prime}$ is induced by a sense-preserving homeomorphism $f: D \rightarrow D$,

(iii) $\delta(j)=\infty$.

Suppose that $j: G \rightarrow G^{\prime}$ satisfying (i)-(iii) is constructed. By Theorem 4.1, there is no quasiconformal self-mapping of $D$ inducing $j$ in $D$ (cf. Theorem 2.1). Moreover, if $\varphi$ is the boundary mapping of $j$, there is no quasiconformal self-mapping of $D$ with boundary values $\varphi$ (Theorem 4.2).

Let $g$ be a hyperbolic transformation fixing $D$, and $I(g)$ its isometric circle (see 3.3). Let $a(g)$ and $c(g)$ denote the points of $I(g) \cap K$ such that $\operatorname{Im}(c(g) / a(g))>0$. If we set $b(g)=A x(g) \cap I(g) \cap D$, then

$$
\varkappa(g)=\left(b\left(g^{-1}\right), b(g), N(g), P(g)\right) .
$$

(The formula (4.13) follows directly from formulae (1.1) since $b\left(g^{-1}\right)=$ $g(b(g))$.

Supposing that $A x(g)$ contains the origin, $g$ is uniquely determined by any two of the numbers $a(g), c(g), \varkappa(g)$ or by $b(g)$ alone.

We define a set $\left\{g_{1}, g_{2}, \ldots\right\}$ of generators of $G$ iductively as follows:

( i) $0 \in A x\left(g_{n}\right), n=1,2, \ldots$,

(ii) $a\left(g_{1}\right)=e^{-i_{\pi} / 4}, c\left(g_{1}\right)=e^{i_{\pi} / 4}$,

(iii) $a\left(g_{n+1}\right)=c\left(g_{n}\right), n=1,2, \ldots$.

(iv) $c\left(g_{n+1}\right)=c\left(g_{n}\right) e^{i_{-7 / 2^{n+1}}}, n=1,2, \ldots$.

Since $c\left(g_{n}\right) \rightarrow g_{1}\left(c\left(g_{1}\right)\right)=a\left(g_{1}^{-1}\right)$ as $n \rightarrow \infty$, it follows that $\left\{g_{1}, g_{2}, \ldots\right\}$ is a free combination. Therefore, $G$ generated by $\left\{g_{1}, g_{2}, \ldots\right\}$ is a covering group (Lemma 3.2). Since the boundary of $F(G)$ contains no arcs of $K$, the group $G$ is of the first kind.

We define a set $\left\{g_{1}^{\prime}, g_{2}^{\prime}, \ldots\right\}$ of generators of $G^{\prime}$ in a slightly different way:

( i) $0 \in A x\left(g_{n}^{\prime}\right), n=1,2, \ldots$,

(ii) $a\left(g_{2}^{\prime}\right)=c\left(g_{1}^{\prime}\right)=e^{i \pi / 4}$,

(iii) $x\left(g_{n}^{\prime}\right)=\varkappa\left(g_{n}\right)^{n}, n=2,3, \ldots$,

(iv) $a\left(g_{n+1}^{\prime}\right)=c\left(g_{n}^{\prime}\right), n=2,3, \ldots$.

(v) $a\left(g_{1}^{\prime}\right)=\lim _{n \rightarrow \infty} c\left(\left(g_{n}^{\prime}\right)^{-1}\right)$. 
It follows from (4.13) that $a\left(g_{1}^{\prime}\right)$ is well defined, i.e., $\operatorname{Im}\left(c\left(g_{1}^{\prime}\right) / a\left(g_{1}^{\prime}\right)\right)>0$, and that $\left\{g_{1}^{\prime}, g_{2}^{\prime}, \ldots\right\}$ is a free combination. Thus $G^{\prime}$ is a covering group of the first kind.

By (ii) in Lemma 3.2, we can define an isomorphism $j: G \rightarrow G^{\prime}$ by $j\left(g_{n}\right)=g_{n}^{\prime}, n=1,2, \ldots$. Then obviously $\delta(j)=\infty$. Moreover, it follows from the construction that there exists a sense-preserving homeomorphism $f_{0}: \mathrm{Bd} F(G) \rightarrow \mathrm{Bd} F\left(G^{\prime}\right)$ such that $f_{0} \circ g_{n}=g_{n}^{\prime} \circ f_{0}, n=$ $1,2, \ldots$. Let $f_{1}: \mathrm{Cl} F(G) \rightarrow \mathrm{Cl} F\left(G^{\prime}\right)$ be an arbitrary homeomorphism with boundary values $f_{1} \mid \operatorname{Bd} F(G)=f_{0}$, and define a homeomorphism $f: D \rightarrow D$ by $f=j(g) \circ f_{1} \circ g^{-1}$ in $g(\mathrm{Cl} F(G) \cap D), g \in G$. Then $f$ induces $j$ in $D$.

\section{§ 5. Isomorphisms with special boundary mappings}

1. In this section all covering groups are of the first kind and act in $H$.

Suppose that $j: G \rightarrow G^{\prime}$ is an isomorphism whose boundary mapping $\varphi$ exists and $\varphi(\infty)=\infty$. We shall first prove that $\varphi$ and $\varphi^{-1}$ are locally Lipschitz mappings if and only if $j$ is induced by a Möbius transformation. We establish this result in a slightly more general form; in fact it suffices to consider only the local behavior of $\varphi$ at the fixed points of $G$. To this end, we give the following definition: $\varphi$ is said to be a Lipschitz mapping at a point $x_{0} \in R$ if there is a real number $L \geq 1$ and a neighborhood $U \subset R$ of $x_{0}$ such that

$$
\left|x_{0}-x\right| / L \leq\left|\varphi\left(x_{0}\right)-\varphi(x)\right| \leq L\left|x_{0}-x\right|
$$

holds for all $x \in U$. (Note that $L$ may depend on $x_{0}$.) This pointwise Lipschitz conditon is evidently invariant under Möbius transformations: if $\varphi$ is a Lipschitz mapping at $x_{0}$ and $h_{1}$ and $h_{2}$ are Möbius transformations fixing $R \cup\{\infty\}$ such that $h_{1}\left(x_{0}\right) \neq \infty$ and $h_{2} \circ \varphi \circ h_{1}^{-1}(\infty)=\infty$, then $h_{2} \circ \varphi \circ h_{1}^{-1}$ is a Lipschitz mapping at $h_{1}\left(x_{0}\right)$. Thus it is reasonable to call $\varphi$ a Lipschitz mapping at $\infty$ if $1 / \varphi(1 / x)$ is a Lipschitz mapping at the origin.

2. We can now establish our proposition.

Theorem 5.1. Let $G$ and $G^{\prime}$ be covering groups of the first kind acting in $H$. An isomorphism $j: G \rightarrow G^{\prime}$ is induced by a Möbius transformation fixing $\infty$ if and only if the boundary mapping $\varphi$ of $j$ is a Lipschitz mapping at the points of Fix $(G)$.

Proof: If $j$ is induced by a Möbius transformation $h$, then $\varphi=$ $h ! R \cup\{\infty\}$. Thus $\varphi$ is a Lipschitz mapping at every point of $R \cup\{\infty\}$. 
Conversely, suppose that $\varphi$ is a Lipschitz mapping at the points of Fix $(G)$. By Theorem 4.3, it suffices to show that $\delta(j)=1$ or, since $\varphi$ exists, that $\varkappa(g)=\varkappa(j(g))$ holds for all hyperbolic transformations $g \in G$. To do this, set $g^{\prime}=j(g)$. Since the pointwise Lipschitz property is invariant under Möbius transformations, we may assume that $P(g)=P\left(g^{\prime}\right)$ $=\infty$. and $N(g)=N\left(g^{\prime}\right)=0$. Choose $x_{0} \in R \backslash\{0\}$ and set $x_{n}=g^{-n}\left(x_{0}\right)$ and $y_{n}=\varphi\left(x_{n}\right)=\left(g^{\prime}\right)^{-n}\left(\varphi\left(x_{0}\right)\right), n=0,1, \ldots$. Then

$$
y_{n} / x_{n}=\left(y_{0} / x_{0}\right)\left(\varkappa(g) / \varkappa\left(g^{\prime}\right)\right)^{n} .
$$

Since $\varphi(0)=0$,

$$
\left|\varphi\left(x_{n}\right)-\varphi(0)\right|=\left|x_{n} y_{0} / x_{0}\right|\left(\varkappa(g) / \varkappa\left(g^{\prime}\right)\right)^{n} .
$$

As $n \rightarrow \infty, x_{n} \rightarrow 0$; thus by (5.1), there is an $L_{0} \geq 1$ such that

$$
1 / L_{0} \leq\left(\varkappa(g) / \varkappa\left(g^{\prime}\right)\right)^{n} \leq L_{0}
$$

holds for all $n=1,2, \ldots$. This implies that $\varkappa(g)=\varkappa\left(g^{\prime}\right)$.

3. Suppose that the boundary mapping $\varphi: \hat{R} \rightarrow \hat{R}$ of an isomorphism $j: G \rightarrow G^{\prime}$ is quasisymmetric. Then there are quasiconformal self-mappings of $H$ with boundary values $\varphi$. Therefore it is natural to ask whether $j$ is induced by a quasiconformal mapping in $H$. We conclude this paper summarizing recent results of Lehto [14] giving a partial answer to this question.

In the following, $G_{0}$ is a fixed covering group of the first kind acting in $H$, and $G$ denotes a similar group that is allowed to vary.

Let $\Omega$ be the set of conformal mappings of the lower half-plane $H^{*}$ onto plane domains. Define $\Delta$ as the set of all $f \in \Omega$ with the following property: there is a quasiconformal mapping $f^{*}: \hat{C} \rightarrow \hat{C}$ such that $f^{*} \mid H^{*}=f$. If $f^{*}$ can be chosen so that $f^{*} \circ g \circ\left(f^{*}\right)^{-1}$ is a Möbius transformation for all $g \in G_{0}$, then $f \in \Delta\left(G_{0}\right)$. Let $Q\left(G_{0}\right)$ be the set of all $f \in \Omega$ such that $f \circ g \circ f^{-1}$ is the restriction of a Möbius transformation for all $g \in G_{0}$. Then $\Delta\left(G_{0}\right) \subset \Delta \cap Q\left(G_{0}\right)$.

Let $J_{h}\left(G_{0}\right)$ be the set of all isomorphisms $j: G_{0} \rightarrow G$ with an increasing boundary mapping $\varphi: \hat{R} \rightarrow \hat{R}, J_{q s}\left(G_{0}\right)$ the set of all $j: G_{0} \rightarrow G$ with a quasisymmetric $\varphi: \hat{R} \rightarrow \hat{R}$, and $J_{q}\left(G_{0}\right)$ the set of all $j: G_{0} \rightarrow G$ induced by quasiconformal self-mappings of $H$ fixing $\infty$. Then $J_{q}\left(G_{0}\right)$ $\subset J_{q s}\left(G_{0}\right) \subset J_{h}\left(G_{0}\right)$.

Theorem 5.2. Let $G_{0}$ be a covering group of the first kind acting in $\mathrm{H}$. Then $J_{q}\left(G_{0}\right)==J_{q s}\left(G_{0}\right)$ if and only if

$$
\Delta\left(G_{0}\right)=\Delta \cap Q\left(G_{0}\right) .
$$


For a proof, see [14]. Kra [12] has proved that (5.2) holds if $G_{0}$ is finitely generated. Thus Theorem 5.2 has the following corollary:

Corollary. Suppose that $G_{0}$ is finitely generated. Then $J_{q}\left(G_{0}\right)=J_{q s}\left(G_{0}\right)$, i.e., an isomorphism $j: G_{0} \rightarrow G$ has a quasisymmetric boundary mapping if and only if $j$ is induced by a quasiconformal self-mapping of $H$ fixing $\infty$.

4. Suppose that $H / G_{0}$ is compact (or, more generally, $G_{0}$ is finitely generated, ef. 4.3). Then by Theorem 2.1, $J_{q}\left(G_{0}\right)=J_{q_{s}}\left(G_{0}\right)=J_{h}\left(G_{0}\right)$. Conversely, if one proves directly that

$$
J_{h}\left(G_{0}\right)=J_{q s}\left(G_{0}\right),
$$

then the above Corollary implies Theorem 2.1.

It can be proved without Nielsen's theorem that $\varphi$ of $j: G_{0} \rightarrow G$ exists whenever $H / G_{0}$ is compact (see [17] p. 240 and Lemma 3.6.2 in $[20])$. Thus Corollary and (5.3) together imply Nielsen's theorem. On the other hand, the proof of (5.2) in [12] involves Nielsen's theorem.

5 . To our knowledge, it is an open question whether (5.2) always holds if $G_{0}$ is not finitely generated (cf. [6]).

Suppose that there is a covering group of the first kind not satisfying (5.2). Then there is an isomorphism $j: G_{0} \rightarrow G$ with a quasisymmetric boundary mapping $\varphi: \hat{R} \rightarrow \hat{R}$ such that $j \notin J_{q}\left(G_{0}\right)$. However, by the following theorem of Lehto [14], the quasisymmetry of $\varphi$ is in this case bounded away from 1 .

Theorem 5.3. If the boundary mapping $\varphi$ of $j \in J_{q s}\left(G_{0}\right)$ satisfies (1.7) with a $\lambda<\sqrt{2}$, then $j \in J_{q}\left(G_{0}\right)$, i.e., $j$ is induced by a quasiconformal self-mapping of $H$.

We remark that the proofs for Theorems 5.2 and 5.3 in [14] do not involve Theorem 3.2.

Department of Mathematics,

University of Helsinki 


\section{References}

[ 1] AGard, S. B.: Topics in the theory of quasiconformal mappings. Ph. D. Thesis. University of Michigan 1965.

[ 2] Ahtfors. L. V.: Finitely generated Kleinian groups. Amer. J. Math. 86 (1964) and $87(1965)$.

[ 3] - - - and Sario, L.: Riemann Surfaces. Princeton University Press, Princeton, N.J., 1960.

[ 4] Bers, L.: Quasiconformal mappings and Teichmüller's theorem. Analytic Function, by R. Nevanlinna, et al. Princeton University Press, Princeton, N.J., 1960.

[5] - $\gg-$ Uniformization by Beltrami equations. Comm. Pure Appl. Math. 14 (1961).

[ 6] -»- Universal Teichmüller space. Analytic Methods in Mathematical Physics, ed. by R. P. Gilbert and R. G. Newton. Gordon and Breach 1970.

[ 7] - - and Greenberg, L.: Isomorphisms between Teichmüller spaces. Advances in the Theory of Riemann Surfaces, by L. V. Ahlfors, et al. Princeton University Press, Princeton, N.J., 1971.

[ 8] Beurling, A. and Ahlfors, L. V.: The boundary correspondence under quasiconformal mappings. Acta Math. 96 (1956).

[ 9] Fenchel, W. and Nielsen, J.: Discontinuous groups of non-Euclidean motions (unpublished).

[10] Ford, L.: Automorphic Functions. Chelsea Publishing Co., New York, 1951.

[11] Hopf, H.: Beiträge zur Klassifizierung der Flächenabbildungen. J. Reine Angew. Math. 165 (1931).

[12] KrA, I.: On Teichmüller spaces for finitely generated Fuchsian groups. Amer. J. Math. 91 (1969).

[13] Lehner, J.: A Short Cource of Automorphic Functions. Holt, Rinehart and Winston, New York, 1966.

[14] Leнto, O.: Group isomorphisms induced by quasiconformal mappings (to appear).

[15] - - und Virtanen, K. I.: Quasikonforme Abbildungen. Springer Verlag, Berlin - Heidelberg - New York, 1965.

[16] Marden, A.: Isomorphisms between Fuchsian groups (unpublished).

[17] Nielsen, J.: Untersuchungen zur Topologie der geschlossenen zweiseitigen Flächen. Acta Math. 50 (1927).

[18] TeichmüLleR, O.: Extremale quasikonforme Abbildungen und quadratische Differentiale. Preuss. Akad. 22 (1940).

[19] - - Bestimmung der extremalen quasikonformen Abbildungen bei geschlossenen orientierten Riemannschen Flächen. Preuss. Akad. 4 (1943).

[20] Tukia, P.: On discrete groups of the unit disk and their isomorphisms. Ann. Acad. Sci. Fenn. AI 504 (1972).

Printed November 1972 